Check for updates

Cite this: Nanoscale Adv., 2019, 1, 834

\title{
Ligand-assisted reduction and reprecipitation synthesis of highly luminescent metal nanoclusters $\dagger$
}

\author{
Yue Wang, ${ }^{\text {ab }}$ Yu-e Shi, ${ }^{\text {ab }}$ Tianzi Li, ${ }^{\mathrm{a}}$ Henggang Wang, ${ }^{\text {a }}$ Yanxiu Li, ${ }^{\mathrm{C}}$ Yuan Xiong, ${ }^{\mathrm{C}}$ \\ Shan Peng (iD ${ }^{a}$ and Zhenguang Wang (D) *ab
}

\begin{abstract}
Solid-state luminescent materials play a key role in fabricating light-emitting diodes (LEDs). Herein, highly luminescent metal nanoclusters (NCs) are synthesized using a ligand-assisted reduction and reprecipitation process. Glutathione (GSH) dissolved in a good solvent (water) is injected into a poor solvent (ethanol in which $\mathrm{Cu}^{2+}$ is dissolved), where the fast reduction of $\mathrm{Cu}^{2+}$ by GSH and the supersaturation-induced aggregation triggered by the solubility change of GSH upon solvent mixing occur. Nanoparticles with diameters of around 50-80 nm embedded with small-sized Cu NCs (around 2 $\mathrm{nm}$ ) can be obtained and processed into powders simply by drying the solvent. The powders show bright-orange emission with a photoluminescence quantum yield as high as $48 \%$. Nearly monoexponential behavior was observed in the photoluminescence decay profiles of the $\mathrm{Cu} N \mathrm{~N}$, which can be attributed to the abundance of metal defect-related states formed with the assistance of coordination between $\mathrm{Cu}$ and ethanol. Moreover, white LEDs were fabricated using blue-emissive commercial phosphors and orange-emissive CU NCs as color converters integrated with UV LED chips.
\end{abstract}

Received 3rd October 2018

Accepted 14th November 2018

DOI: $10.1039 / \mathrm{c} 8 \mathrm{na00268a}$

rsc.li/nanoscale-advances toxic precursors as substitutes for heavy metal- and rare earthbased materials is an important research topic.

Metal nanoclusters (NCs), nano-sized metal atom cores surrounded by ligands, have emerged as promising materials due to their remarkable chemical and optical properties, including earth abundance, non-toxicity, HOMO-LUMO transition and reasonably high PL. ${ }^{15-19}$ However, highly luminescent metal NC-based SLMs are difficult to realize because solid-state metal NCs are prone to becoming highly concentrated or even aggregated. This leads to the deterioration of their PL properties as a result of reabsorption and nonradiative energy transfer, which is known as aggregation-caused quenching. The concept of aggregation-induced emission (AIE), coined by Tang's group, provides new perspectives for designing highly luminescent SLMs that avoid aggregation-caused quenching. ${ }^{20,21} \mathrm{We}$ and other groups have reported SLMs using the AIE properties of $\mathrm{Cu}$ and $\mathrm{Au} \mathrm{NCs.} .^{22-26}$ The enhanced emission can be ascribed to two mechanisms resulting from the promoted compactness of metal NCs: (a) enhancing the inter- and intra-NC metallophilic interactions by dealing with the metal defects on the metal NC surfaces; and (b) reducing the nonradiative relaxation of excited states by restricting the vibrations and rotations of ligands. ${ }^{20,26}$ To achieve high compactness, an excess of poor solvent is usually mixed with the NCs in a small amount of good solvent to precipitate the luminescent materials. The aforementioned solvents are then discarded. On one hand, this operation is complicated and leads to extremely diluted NCs, making it difficult to obtain SLMs on a large scale. 
On the other hand, aggregation is driven by the difference in the ligand's solubility in good and poor solvents, which can effectively restrict the vibrations and rotations of ligands. However, the metal defects cannot be well controlled or even passivated by the excess of poor solvent.

Herein, a straightforward method (Fig. 1a) for the gram-scale synthesis of luminescent $\mathrm{Cu}$ NCs is developed by using a ligandassisted reduction and reprecipitation (LARR) process to control the compactness of the $\mathrm{Cu}$ NCs. This process both enhances the metallophilic interactions and restricts the vibrations and rotations of ligands. The $\mathrm{Cu}$ NCs can then be used to produce SLMs. Glutathione (GSH) is selected as the ligand to reduce $\mathrm{Cu}^{2+}$ and trigger the aggregation of $\mathrm{Cu}$ clusters based on its difference in solubility in good and poor solvents, as confirmed by comparative studies of different ligands along with optical and structural characterizations. The PL mechanisms are studied by recording the PL decay curves of the $\mathrm{Cu}$ NCs at different emission wavelengths. We further demonstrated the application of the Cu NCs in the fabrication of LEDs by using the $\mathrm{Cu}$ NCs as light-conversion layers.

\section{Results and discussion}

\section{Synthesis of $\mathrm{Cu}$ NCs via the LARR process}

The LARR synthesis is accomplished simply by injecting good solvent (water) containing GSH into poor solvent (ethanol into which $\mathrm{Cu}^{2+}$ is dissolved) under vigorous stirring, as shown in Fig. 1a. Typically, $0.5 \mathrm{~mL}$ of saturated aqueous GSH solution is quickly injected into $9.5 \mathrm{~mL}$ of ethanol solution containing $5 \mathrm{mM} \mathrm{Cu}{ }^{2+}$ under vigorous stirring. A dark black color is observed immediately upon injection and gradually turns into a milky white color, signaling the formation of $\mathrm{Cu}$ NCs. GSH (structure shown in Fig. 1b) acts as both a reduction reagent to lower the valence of $\mathrm{Cu}^{2+}$ and a trigger for aggregation based on its huge difference in solubility between water and ethanol. The solution is centrifuged at $500 \mathrm{krpm}$ to remove the large yellow particles, and the supernatant solution is collected. The supernatant shows strong orange emission under UV

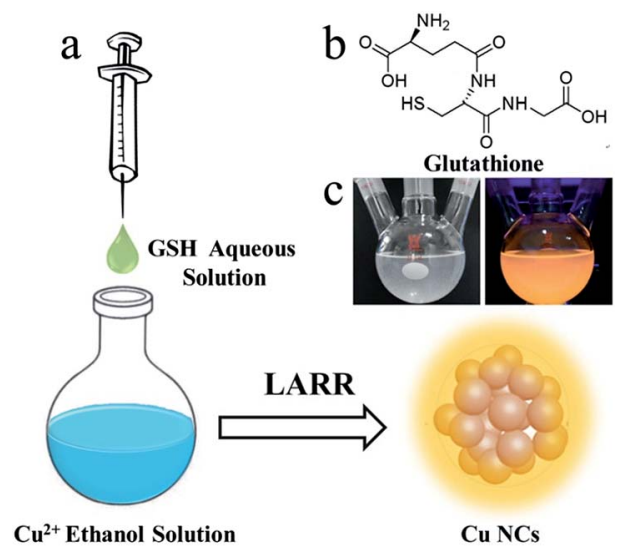

Fig. 1 (a) Schematic illustration of the synthesis of Cu NCs using the LARR method. (b) Structure of GSH. (c) Optical images of a solution of Cu NCs under daylight and UV light. irradiation, as shown in Fig. 1c. To understand the role of GSH in the synthetic process, contrast experiments are carried out. Ligands with structures similar to GSH, including cysteine and cysteamine, were used to synthesize Cu NCs. Upon injecting cysteamine into ethanol containing $\mathrm{Cu}^{2+}$, the deep-blue solution immediately turns colorless. Upon cysteine injection, the solution becomes black. The solutions become cloudy after stirring for $5 \mathrm{~min}$, indicating the reduction of $\mathrm{Cu}^{2+}$ by the above two ligands. However, no emission under UV irradiation is observed for the $\mathrm{Cu}$ NCs synthesized using the above ligands. This can be explained by the fact that cysteine and cysteamine are soluble in ethanol; thus, dense aggregates of $\mathrm{Cu}$ NCs and subsequent AIE effects are not observed. Based on the above observations, we conclude that the formation of $\mathrm{Cu}$ NCs is controlled by the fast reduction of $\mathrm{Cu}$ ions by GSH and the supersaturation-induced aggregation based on the solubility change of GSH upon solvent mixing.

\section{Characterization of $\mathrm{Cu}$ NCs}

Fig. 2a shows typical transmission electron microscopy (TEM) images of the $\mathrm{Cu}$ NCs. Quasi-spherical nanoparticles with diameters in the range of 50 to $80 \mathrm{~nm}$ can be recognized (Fig. 2a), suggesting that the as-synthesized samples are monodisperse. Within a certain nanoparticle, small-sized $\mathrm{Cu}$ NCs with an average diameter of $2.3 \mathrm{~nm}$ are embedded rather than crystalline $\mathrm{Cu}$. The structure is formed during the ligandassisted reduction and reprecipitation process, in which the fast reduction of $\mathrm{Cu}^{2+}$ by $\mathrm{GSH}$ and the supersaturation-induced aggregation triggered by the change in GSH solubility upon solvent mixing occur. The $\mathrm{Cu}$ NCs are synthesized and selfassemble within a single nanoparticle, which is driven by the difference in $\mathrm{Cu}$ NC solubility in good (water) and poor (ethanol) solvent. The XRD spectrum (Fig. S1†), which shows only a broad peak at around $20^{\circ}$, confirms the poor crystallizability of the $\mathrm{Cu}$ NCs. The composition of the $\mathrm{Cu}$ NCs was studied by XPS, and all the expected constituent elements of the
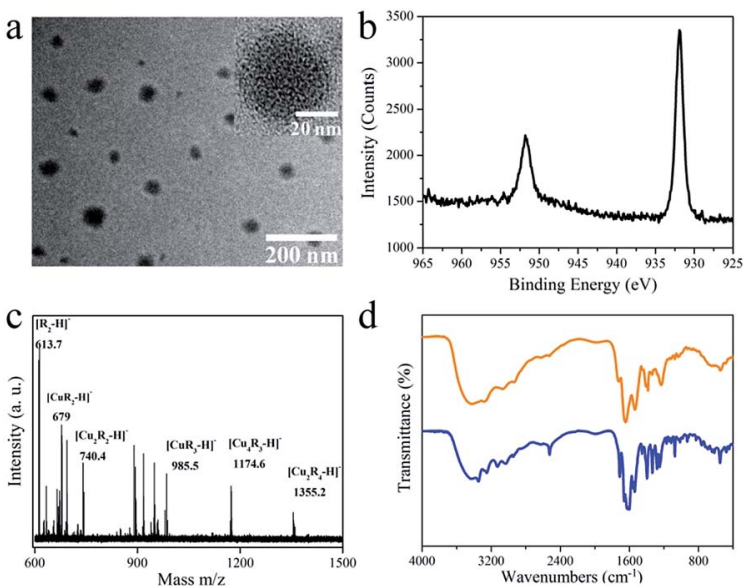

Fig. 2 Characterization of $\mathrm{Cu}$ NCs. (a) TEM image (inset shows the HRTEM image). (b) XPS spectrum of $\mathrm{Cu} 2 \mathrm{p}$ electrons. (c) MALDI-TOF MS spectrum of Cu NCs. (d) FTIR spectra of Cu NCs (orange line) and GSH (blue line) 
composite (C, O, N, S and $\mathrm{Cu}$ ) were detected (Fig. S2 $\dagger$ ). To study the valence state of $\mathrm{Cu}$, the $\mathrm{Cu} 2 \mathrm{p}$ XPS spectrum was recorded (Fig. 2b). The reduction of $\mathrm{Cu}$ ions by GSH is demonstrated by the absence of typical $\mathrm{Cu}^{2+}$ peaks around $942 \mathrm{eV}$. The two intense peaks around 932 and $952 \mathrm{eV}$ belong to the $2 \mathrm{p}_{1 / 2}$ and $2 \mathrm{p}_{2 / 3}$ electrons of metallic copper. ${ }^{27-29}$ The valence of $\mathrm{Cu}$ lies between 0 and +1 , with $\mathrm{Cu}$ atom in the core and $\mathrm{Cu}^{+}$located at the surface or bound to the ligands. To determine the ratio of $\mathrm{Cu}$ to GSH in the as-synthesized Cu NCs, XPS and TGA were conducted. The molecular ratio of $\mathrm{Cu}$ to $\mathrm{S}$ was determined to be $3: 1$ based on the XPS results. As shown in the TGA curve of the $\mathrm{Cu}$ NCs (Fig. S3†), the $\mathrm{Cu}$ NCs are thermally stable in the temperature range of $30^{\circ} \mathrm{C}$ to $185^{\circ} \mathrm{C}$, with more than $96 \%$ of the weight preserved. A sharp decrease in weight is observed in the temperature range of $200{ }^{\circ} \mathrm{C}$ to $400{ }^{\circ} \mathrm{C}$. This weight loss is attributed to the decomposition of GSH ligands. Only $41 \%$ of the weight is retained after heating to $800{ }^{\circ} \mathrm{C}$. The ratio of $\mathrm{Cu}$ to GSH was calculated to be $\sim 3.2$, which is consistent with the XPS measurements. MALDI-TOF MS analysis was carried out to determine the composition of the $\mathrm{Cu}$ NCs (Fig. 2c). The two major peaks located at $m / z=1355.2$ and 1174.7 in Fig. 2c correspond to $\left[\mathrm{Cu}_{2} \mathrm{R}_{4}{ }^{-} \mathrm{H}\right]^{-}$and $\left[\mathrm{Cu}_{3} \mathrm{R}_{3}{ }^{-} \mathrm{H}\right]^{-}$, respectively, where $\mathrm{R}=\mathrm{C}_{10} \mathrm{H}_{16} \mathrm{O}_{6} \mathrm{~N}_{3} \mathrm{~S}$. FTIR was further used to study the interaction between GSH and the Cu NCs (Fig. 2d). No other new or obliterated peaks are observed in the FTIR spectra of GSH and $\mathrm{Cu}$ NCs, except for the disappearance of the peak at $2524 \mathrm{~cm}^{-1}$, which corresponds to the S-H stretching vibration mode of $\mathrm{GSH}^{30}$ This indicates that GSH is connected to the Cu NCs via $\mathrm{Cu}-\mathrm{S}$ bonds without connections with other chemical groups, such as $-\mathrm{NH}_{2}$ and $-\mathrm{COOH}$. The above results strongly support our hypothesis that nanoparticles containing small-sized $\mathrm{Cu}$ NCs are synthesized via the GSH-assisted reduction of $\mathrm{Cu}^{2+}$ and reprecipitation controlled by the injection of GSH dissolved in good solvent into poor solvent.

\section{PL properties of the Cu NCs}

Fig. 3a shows the UV-visible absorption spectrum of the assynthesized $\mathrm{Cu}$ NCs. The spectrum is rather structure-less with a vague peak around $365 \mathrm{~nm}$. This peak can be ascribed to the inter-band electronic transitions of the $\mathrm{Cu}$ NCs. The absence of surface plasmon resonance peaks around 560$600 \mathrm{~nm}$ excludes the formation of large copper particles..$^{30}$ As shown in Fig. 3a, an intense orange emission peak at $615 \mathrm{~nm}$ and a PL excitation (PLE) peak around $350 \mathrm{~nm}$ were recorded, resulting in a PL QY as high as $29.7 \%$. This QY is higher than those of most reported $\mathrm{Cu}$ NCs and better than that of $\mathrm{Cu}$ NCs synthesized through reprecipitation by directly injecting $\mathrm{Cu}$ NCs into poor solvents. ${ }^{12}$ The as-synthesized Cu NCs can be feasibly processed into solid sates by evaporating the solvent, producing luminescent NC powders (inset of Fig. 3a). It is worth noting that a blue shift from 615 to $600 \mathrm{~nm}$ is observed in the peak in the PL spectra of the Cu NCs in solution and powder states. After processing into the power state, the average interatomic distance can be increased by increasing the ratio of inter-NC metallophilic interactions to intrinsic intra-NC metallophilic interactions. This leads to the promotion of emission

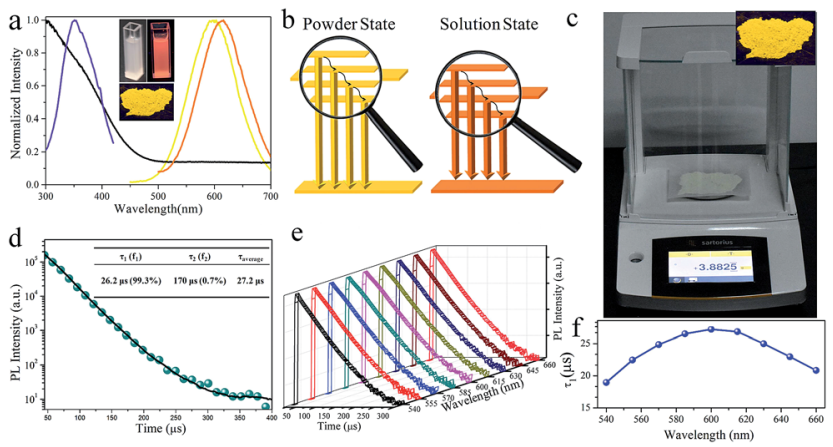

Fig. 3 (a) UV-visible absorption (black line), PL (orange line) and PLE (purple line) spectra of the as-synthesized Cu NCs, PL spectrum of $\mathrm{Cu}$ NC powder (yellow line), and photos under irradiation by daylight and UV light. (b) Schematic Illustration of the energy level of Cu NCs with powder and solution state; (c) photos of sample (more than $3 \mathrm{~g}$ ) obtained from large-scale synthesis and its photo showing orange emission under UV radiation (insert); (d) PL decay profiles of Cu NC powder, showing a nearly monoexponential decay, with PL lifetimes $\left(\tau_{1-2}, \mu s\right)$ and fractions $\left(f_{1-2}, \%\right)$ obtained from the fitting of bioexperimental data shown in the inset; (e) PL decay profiles and (f) PL lifetimes of Cu NC powder detected at different emission wavelength.

energy and a blue shift in the emission peak of the Cu NCs, as depicted in Fig. 3b. The LARR synthesis can easily produce grams of orange-emissive solid materials with PL QY as high as $48 \%$ through batch experiments simply by scaling up the synthesis (Fig. 3c). As stated in the introduction, the AIE can be ascribed to the enhanced NC metallophilic interactions and the restriction of ligand vibration and rotation. Similar to most of the reported AIE studies, using overdosed poor solvents to trigger the AIE of metal NCs, the aggregation of Cu NCs caused by the supersaturation of ligands hinders the flexibility of GSH on the surface of the clusters, which results in the suppression of non-radiative decay channels and promoted PL QY. Moreover, metal defects in the $\mathrm{Cu}$ NCs are deliberately created during the LARR process. Before reaction, $\mathrm{Cu}^{2+}$ is dissolved into ethanol and allowed to coordinate with $\mathrm{Cu}$, which alters the coordination environment of $\mathrm{Cu}$ atoms and the surface properties of $\mathrm{Cu}$ clusters. ${ }^{25}$ This may create more metal detects and prevent their passivation during synthesis, which allows more exited electrons to be transferred to a metal defect-related intermediate state, leading to a PL QY higher than that of aggregated metal NCs prepared by simply dispersing clusters in excess poor solvent. Our results are in agreement with $\mathrm{Wu}$ and co-authors, who found that the PL QY can be tuned by adjusting the number of metal defects on $\mathrm{Cu}$ NCs. ${ }^{25}$

\section{Nearly monoexponential PL decay behavior of $\mathrm{Cu}$ NCs}

To gain more insight into the dynamics of exciton recombination, the PL decay curves of the Cu NCs were measured (Fig. 3d). The decay curves can be fit biexponentially with a shorter PL lifetime $\left(\tau_{1}\right)$ of $26.2 \mu \mathrm{s}(99.3 \%)$ and a longer PL lifetime $\left(\tau_{2}\right)$ of $170 \mu \mathrm{s}(0.7 \%)$. The biexponential decay behavior indicates that two species are involved in the emission of the Cu NCs. In light of the study of the mechanisms of metal NCs with AIE 
phenomena, metal defect-related emissions are the main contributors to the improved PL QY. Compared to reported metal NCs, the decay of the $\mathrm{Cu}$ NCs shows a nearly monoexponential behavior, with $\tau_{1}$ accounting for more than $99 \%$ of the decay, which can be related to exciton recombination from metal defect-related emission. Monoexponential decay often indicates that radiative processes are dominant, and a PL QY of unity is expected to be achieved. ${ }^{31}$ The PL QY of the Cu NCs is only $48 \%$; thus, around $50 \%$ of the clusters are assumed to be located in dark states because they failed to relax into the metal defect-related states. The PL decay of $\mathrm{Cu}$ NCs at different emission wavelengths were recorded and are shown in Fig. 3e. Nearly monoexponential behavior is observed at different detection wavelengths, with $\tau_{1}$ accounting for more than $99 \%$ of decay, as shown in Table S1. $\uparrow$ This indicates that all emissions are from the same metal defect-related state. The PL lifetimes of $\tau_{1}$ detected at different wavelengths are presented in Fig. 3f, indicating wavelength-dependent properties. This indicates the trapping of multiple electrons during the recombination process of the $\mathrm{Cu}$ NCs, as indicated schematically in Fig. 3b. A maximum value of $\tau_{1}$ is obtained at the wavelength of $600 \mathrm{~nm}$, the same as the position of the peak in the PL spectrum. This suggests that emission from the peak corresponds to the lowest energy level of the metal defect-related states. ${ }^{26}$

\section{Applications for LEDs}

The luminescent powder with high PL QY and potential for large-scale synthesis provides an opportunity to explore its application in LEDs. The toxicity of $\mathrm{Cu}$ NCs is an important consideration for practical applications. In this study, Cu NC cytotoxicity was evaluated by MTS assay in 3T3 fibroblasts. As shown in Fig. S4, $\uparrow$ only a slight decrease in cell viability is observed after incubation for $24 \mathrm{~h}$, and over $95 \%$ cell viability is retained by cells incubated with $\mathrm{Cu}$ NCs at concentrations as high as $12 \mu \mathrm{g} \mathrm{mL}^{-1}$. This suggests that the as-synthesized $\mathrm{Cu}$ NCs have low toxicity. To demonstrate potential illumination applications, white LEDs were fabricated by combing blueemissive commercial phosphors (BMA) and orange-emissive $\mathrm{Cu}$ NCs with UV-LED chips. Fig. 4a shows photographs of the blue- and orange-emitting powders taken under UV illumination together with their PL and PLE spectra. The emission of the blue and orange powders covers the full visible spectral range from 400 to $700 \mathrm{~nm}$, demonstrating the potential to generate white light. Moreover, a wide overlap (from 300 to $400 \mathrm{~nm}$ ) is observed in the PLE spectrum; thus, both phosphors can be excited using a single UV light. Blue, orange and white LEDs were fabricated by integrating BMA, Cu NCs and a mixture of both with UV-LED chips (emitting light centered at $370 \mathrm{~nm}$, as shown in Fig. S5†). Fig. $4 \mathrm{~b}$ and $\mathrm{c}-\mathrm{f}$ show photographs of the devices and the emission spectra of blue, orange and white LEDs, respectively. Moreover, the Commission Internationale de L'Eclairage (CIE) chromaticity positions of the LEDs can be tuned form blue $(0.19,0.14)$ to white light $(0.33,0.32)$ towards orange $(0.45,0.48)$ by systematically controlling the weight ratio of blue- to orange-emitting phosphors. After optimization, white LEDs with CIE chromaticity coordinates of $(0.33,0.32)$,
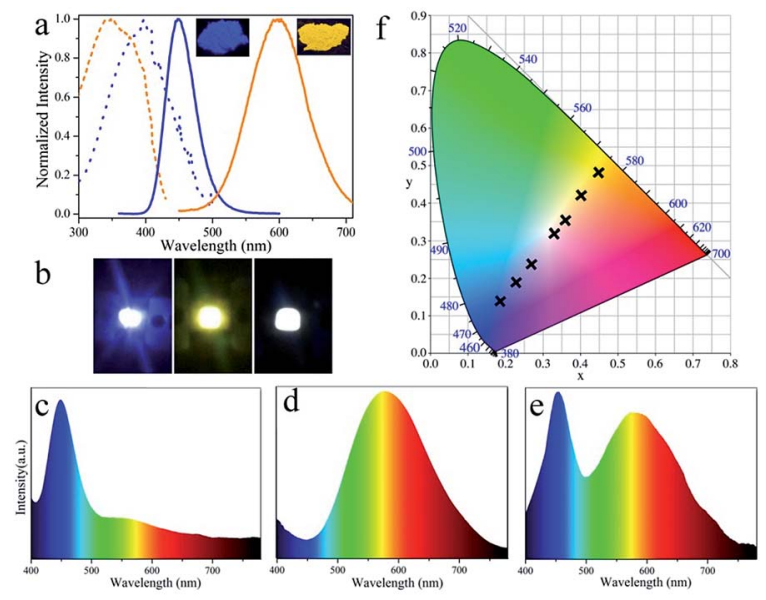

Fig. 4 (a) Optical spectra of phosphors with photographs under UV illumination shown as insets: PL (solid line, excited at $365 \mathrm{~nm}$ ) and PLE (dotted line, detected at the corresponding PL peaks) of BMA (blue) and Cu NCs (orange). (b) Photographs of operating blue, orange and white LEDs. (c-e) Emission spectra of blue, orange and white LEDs, respectively. (f) $\mathrm{CIE}$ chromaticity coordinate diagram of LEDs fabricated by mixing different ratios of blue and orange phosphors.

close to sunlight, ${ }^{32}$ a high CRI of 89 , and a color temperature of $4519 \mathrm{~K}$ were fabricated.

\section{Conclusions}

In summary, a straightforward LARR method for synthesizing brightly luminescent $\mathrm{Cu}$ NCs is proposed. This method is low in cost, avoids the use of toxic metals or rare earth elements, and can be conveniently scaled up for large-scale synthesis. The assynthesized $\mathrm{Cu}$ NCs show a PL QY as high as $48 \%$, which is attributed to the AIE effect caused by supersaturation-induced aggregation and the protection of metal defects by coordination between ethanol and $\mathrm{Cu}$. Moreover, the PL decay curves show nearly monoexponential behavior due to the existence of metal defect-related states. The homogeneity of PL decay behavior at different detection wavelengths and the wavelengthdependent PL lifetimes demonstrate that the emissions of the $\mathrm{Cu}$ NCs come from the same metal defect-related state, and multiple electrons are trapped during the recombination process. After integrating the orange-emissive $\mathrm{Cu}$ NCs and blueemissive phosphors with UV-LED chips, we developed a series of LEDs with tunable emission color from blue to white to orange. A white light with favorable CIE coordinates $(0.33,0.32)$, CRI (89) and color temperature (4519 K) is achieved. The availability of luminescent $\mathrm{Cu}$ NCs adds a new family member to SLMs and will open the door to numerous applications in sensing, bioimaging, security printing and LEDs.

\section{Experimental}

\section{Materials}

Chemicals including copper(II) nitrate $\left[\mathrm{Cu}\left(\mathrm{NO}_{3}\right)_{2}\right]$ and $\mathrm{GSH}$ were purchased from Sigma-Aldrich, USA. L-Cysteine hydrochloride anhydrous and cysteamine were purchased from Kermel, 
Tianjin, China. Commercial blue phosphors $\left(\mathrm{BaMgAl}_{10} \mathrm{O}_{7}: \mathrm{Eu}\right.$, BMA) were obtained from Shenzhen Looking Long technology Co. Ltd. Silicone resin components OE-6551A and OE-6551B were purchased from Dow Corning Co.

\section{Synthesis of Cu NCs}

$\mathrm{Cu}$ NCs were synthesized through a LARR process. Typically, $0.5 \mathrm{~mL}$ of $\mathrm{GSH}$ aqueous solution $(250 \mathrm{mM})$ was injected into $9.5 \mathrm{~mL}$ ethanol solution containing $5 \mathrm{mM}$ dissolved $\mathrm{Cu}^{2+}$ under vigorous stirring. The color of the mixture changed from intense blue to black to dark green and finally to milky as the stirring time increased. Finally, the products were centrifuged at $500 \mathrm{krpm}$ to remove the large yellow particles, and the supernatant solution was collected. $\mathrm{Cu}$ NC powder was prepared by discarding the solvent and drying in an oven.

\section{Fabrication of LEDs}

To fabricate blue or orange LEDs, $0.1 \mathrm{~g}$ of blue phosphor or $\mathrm{Cu}$ NC powder was mixed with $0.1 \mathrm{~g}$ of silicone resin OE-6551A. To fabricate WLEDs, $0.1 \mathrm{~g}$ of blue phosphor and $0.4 \mathrm{~g} \mathrm{Cu} \mathrm{NC}$ powder were mixed with $0.1 \mathrm{~g}$ of OE-6551A. The above mixtures were then dried at $50{ }^{\circ} \mathrm{C}$ for $1 \mathrm{~h}$ and mixed with $0.2 \mathrm{~g} \mathrm{OE}-6551 \mathrm{~B}$ powder. After coating onto GaN LED chips, the emission wavelength peaked at $370 \mathrm{~nm}$.

\section{Characterization}

UV-visible and PL spectra were collected using a Varian Cary 50 UV-visible spectrophotometer and a Varian Cary Eclipse fluorescence spectrometer, respectively. TEM images were recorded on a Philips CM 20 microscope. XPS measurements of Cu NCs were conducted on an ESCALAB-MKII 250 photoelectron spectrometer (Thermo, USA). MALDI-TOF MS spectra were recorded using a mass spectrometer (Bruker Daltonics, USA). FTIR spectra of GSH and Cu NCs were recorded on a Perkin-Elmer Spectrum 100 FTIR spectrometer. Time-resolved PL decay curves were collected on a time-correlated single-photon counting setup under excitation with a $320 \mathrm{~nm}$ laser. PL decay curves were fitted with two-exponential functions in the form of eqn (1):

$$
I(t)=B_{1} \mathrm{e}^{-\frac{t}{\tau_{1}}}+B_{2} \mathrm{e}^{-\frac{t}{\tau_{2}}}, B_{1}+B_{2}=1,
$$

where $\tau_{1}$ and $\tau_{2}$ represent the decay constants, and $B_{1}$ and $B_{2}$ represent the normalized amplitudes of each component. The amplitude-weighted average lifetime of the entire photoluminescence decay process was calculated using eqn (2):

$$
\tau=\frac{B_{1} \tau_{1}^{2}+B_{2} \tau_{2}^{2}}{B_{1} \tau_{1}+B_{2} \tau_{2}} .
$$

Absolute PL QY was determined using a spectrofluorometer (FLS920P, Edinburgh Instruments) equipped with an integrating sphere. The LED parameters, including CIE color coordinates, CRI, and color temperature, were recorded using a high-accuracy array rapid spectroradiometer (Haas-2000, Everfine Co., Ltd., China) equipped with an integrating sphere.

\section{Conflicts of interest}

There are no conflicts to declare.

\section{Acknowledgements}

This work is supported by the National Natural Science Foundation of China (21804030).

\section{References}

1 C.-L. Liu, R.-L. Zhang, C.-S. Lin, L.-P. Zhou, L.-X. Cai, J.-T. Kong, S.-Q. Yang, K.-L. Han and Q.-F. Sun, J. Am. Chem. Soc., 2017, 139, 12474-12479.

2 W. Guan, W. Zhou, J. Lu and C. Lu, Chem. Soc. Rev., 2015, 44, 6981-7009.

3 A. Fernández and M. Vendrell, Chem. Soc. Rev., 2016, 45, 1182-1196.

4 G. G. Dias, A. King, F. de Moliner, M. Vendrell and E. N. da Silva Júnior, Chem. Soc. Rev., 2018, 47, 12-27.

5 H. Sun, S. Liu, W. Lin, K. Y. Zhang, W. Lv, X. Huang, F. Huo, H. Yang, G. Jenkins, Q. Zhao and W. Huang, Nat. Commun., 2014, 5, 3601.

6 K. Jiang, Y. Wang, C. Cai and H. Lin, Adv. Mater., 2018, 30, 1800783.

7 J. Lee, H.-F. Chen, T. Batagoda, C. Coburn, P. I. Djurovich, M. E. Thompson and S. R. Forrest, Nat. Mater., 2015, 15, 92.

8 Z. Wang, F. Yuan, X. Li, Y. Li, H. Zhong, L. Fan and S. Yang, Adv. Mater., 2017, 29, 1702910.

9 H. V. Demir, S. Nizamoglu, T. Erdem, E. Mutlugun, N. Gaponik and A. Eychmüller, Nano Today, 2011, 6, 632-647.

10 H. Terraschke and C. Wickleder, Chem. Rev., 2015, 115, 11352-11378.

11 L. Wang, R.-J. Xie, T. Suehiro, T. Takeda and N. Hirosaki, Chem. Rev., 2018, 118, 1951-2009.

12 Z. Wang, B. Chen, A. S. Susha, W. Wang, C. J. Reckmeier, R. Chen, H. Zhong and A. L. Rogach, Adv. Sci., 2016, 3, 1600182.

13 Z. Wang, Q. Jingjing, X. Wang, Z. Zhang, Y. Chen, X. Huang and W. Huang, Chem. Soc. Rev., 2018, 47, 6128-6174.

14 L. Jing, S. V. Kershaw, Y. Li, X. Huang, Y. Li, A. L. Rogach and M. Gao, Chem. Rev., 2016, 116, 10623-10730.

15 Q. Yao, T. Chen, X. Yuan and J. Xie, Acc. Chem. Res., 2018, 51, 1338-1348.

16 Q. Yao, V. Fung, C. Sun, S. Huang, T. Chen, D.-e. Jiang, J. Y. Lee and J. Xie, Nat. Commun., 2018, 9, 1979.

17 X. Kang, M. Zhou, S. Wang, S. Jin, G. Sun, M. Zhu and R. Jin, Chem. Sci., 2017, 8, 2581-2587.

18 T. Higaki, C. Liu, M. Zhou, T.-Y. Luo, N. L. Rosi and R. Jin, J. Am. Chem. Soc., 2017, 139, 9994-10001.

19 I. Chakraborty and T. Pradeep, Chem. Rev., 2017, 117, 82088271.

20 J. Mei, N. L. C. Leung, R. T. K. Kwok, J. W. Y. Lam and B. Z. Tang, Chem. Rev., 2015, 115, 11718-11940.

21 J. Luo, Z. Xie, J. W. Y. Lam, L. Cheng, H. Chen, C. Qiu, H. S. Kwok, X. Zhan, Y. Liu, D. Zhu and B. Z. Tang, Chem. Commun., 2001, 1740-1741. 
22 Z. Wang, Y. Xiong, S. V. Kershaw, B. Chen, X. Yang, N. Goswami, W.-F. Lai, J. Xie and A. L. Rogach, Chem. Mater., 2017, 29, 10206-10211.

23 Z. Luo, X. Yuan, Y. Yu, Q. Zhang, D. T. Leong, J. Y. Lee and J. Xie, J. Am. Chem. Soc., 2012, 134, 16662-16670.

24 X. Jia, J. Li and E. Wang, Small, 2013, 9, 3873-3879.

25 Z. Wu, H. Liu, T. Li, J. Liu, J. Yin, O. F. Mohammed, O. M. Bakr, Y. Liu, B. Yang and H. Zhang, J. Am. Chem. Soc., 2017, 139, 4318-4321.

26 Y. Liu, D. Yao and H. Zhang, ACS Appl. Mater. Interfaces, 2018, 10, 12071-12080.
27 Z. Wang, B. Chen and A. L. Rogach, Nanoscale Horiz., 2017, 2, 135-146.

28 Z. Wang, Y.-e. Shi, X. Yang, Y. Xiong, Y. Li, B. Chen, W.-F. Lai and A. L. Rogach, Adv. Funct. Mater., 2018, 28, 1802848.

29 Z. Wu, J. Liu, Y. Gao, H. Liu, T. Li, H. Zou, Z. Wang, K. Zhang, Y. Wang, H. Zhang and B. Yang, J. Am. Chem. Soc., 2015, 137, 12906-12913.

30 X. Liu and D. Astruc, Coord. Chem. Rev., 2018, 359, 112-126. 31 S. F. Wuister, I. Swart, F. van Driel, S. G. Hickey and C. de Mello Donegá, Nano Lett., 2003, 3, 503-507.

32 C. C. Lin, A. Meijerink and R.-S. Liu, J. Phys. Chem. Lett., 2016, 7, 495-503. 